\title{
Primitive lattice points in convex planar domains
}

\author{
by
}

\section{Martin N. Huxley (Cardiff) and Werner Georg Nowak (Vienna)}

1. Introduction. Let $\mathcal{D}$ denote a compact convex subset of $\mathbb{R}^{2}$ which contains the origin as an inner point. Suppose that the boundary $\partial \mathcal{D}$ of $\mathcal{D}$ is smooth with finite nonzero curvature throughout, and define a "canonical map" $\mathcal{M}$ from $\partial \mathcal{D}$ to the unit circle, which maps every point $\mathbf{u}$ of $\partial \mathcal{D}$ to the outward normal vector of $\partial \mathcal{D}$ in $\mathbf{u}$ of length unity. Assume that $\mathcal{M}$ is one-one and of class $\left(^{1}\right) C^{\infty}$. Let $F$ denote the distance function of $\mathcal{D}$, i.e.,

$$
F(\mathbf{u})=\inf \{\tau>0: \mathbf{u} / \tau \in \mathcal{D}\} \quad\left(\mathbf{u} \in \mathbb{R}^{2}\right),
$$

and put $Q=F^{2}$, thus $Q$ is homogeneous of degree 2 . For a large real variable $x$, define $A_{\mathcal{D}}(x)$ as the number of lattice points of $\mathbb{Z}_{*}^{2}:=\mathbb{Z}^{2} \backslash\{(0,0)\}$ in the "blown up" domain $\sqrt{x} \mathcal{D}$, i.e.,

$$
A_{\mathcal{D}}(x)=\#\left(\sqrt{x} \mathcal{D} \cap \mathbb{Z}_{*}^{2}\right)=\#\left\{\mathbf{m} \in \mathbb{Z}_{*}^{2}: Q(\mathbf{m}) \leq x\right\},
$$

and $P_{\mathcal{D}}(x)$ as the "lattice rest"

$$
P_{\mathcal{D}}(x)=A_{\mathcal{D}}(x)-a(\mathcal{D}) x,
$$

where $a(\mathcal{D})$ is the area of $\mathcal{D}$.

Only recently, Huxley [9], Theorem 5, showed that the problem of estimating $P_{\mathcal{D}}(x)$ from above is not more difficult for general $\mathcal{D}$ than for the classic case that $\mathcal{D}$ is the unit disk. He proved that

$$
P_{\mathcal{D}}(x)=O\left(x^{23 / 73}(\log x)^{315 / 146}\right),
$$

uniformly with respect to rotations and translations of $\sqrt{x} \mathcal{D}$.

1991 Mathematics Subject Classification: 11P21, 11M26, $11 \mathrm{M} 41$.

Key words and phrases: primitive lattice points, Riemann Hypothesis.

This article is part of a research project supported by the Austrian Science Foundation (Nr. P 9892-PHY).

$\left(^{1}\right)$ In this supposition, $C^{\infty}$ can be replaced by $C^{K}$ with some $K \in \mathbb{N}$ sufficiently large. The whole somewhat technical condition is only stated to ensure the validity of the estimate (1.3), which in turn depends on the asymptotic expansion for the Fourier coefficients of the indicator function of $\sqrt{x} \mathcal{D}$ (see Hlawka [6], [7]). 

that

A bit earlier, the second named author [17], [18] had obtained the results

$$
\lim _{x \rightarrow \infty} \inf \left(\frac{P_{\mathcal{D}}(x)}{(x \log x)^{1 / 4}}\right)<0,
$$

and

$$
\int_{0}^{T}\left(P_{\mathcal{D}}(x)\right)^{2} d x \ll T^{3 / 2}, \quad \int_{0}^{T}\left|P_{\mathcal{D}}(x)\right| d x \ll T^{5 / 4} .
$$

Refined mean-square results, with somewhat relaxed smoothness conditions on the boundary of $\mathcal{D}$, may be found in the first named author's paper [10] and in his monograph [11].

For the classic circle problem (cf., e.g., the book of Krätzel [14] for its history), a slight improvement of (1.2) has been established by Hafner [3], while the mean-square bound in (1.3) may be replaced by an asymptotic formula: See Kátai [13] for the sharpest version to date.

The objective of the present paper is to study the number $B_{\mathcal{D}}(x)$ of primitive lattice points in $\sqrt{x} \mathcal{D}$, i.e.,

$$
B_{\mathcal{D}}(x)=\#\left\{\mathbf{u}=\left(u_{1}, u_{2}\right) \in \mathbb{Z}_{*}^{2}: Q(\mathbf{u}) \leq x, \operatorname{gcd}\left(u_{1}, u_{2}\right)=1\right\} .
$$

By a usual device (which is sometimes attributed to Vinogradov),

$$
B_{\mathcal{D}}(x)=\sum_{m \in \mathbb{N}} \mu(m) A_{\mathcal{D}}\left(\frac{x}{m^{2}}\right),
$$

where $\mu(m)$ denotes the Möbius function. By an elementary convolution argument, one can derive from the bound

$$
\sum_{m \leq Y} \mu(m) \ll Y \omega(Y)
$$

(see Ivić [12], p. 309), combined with (1.4) and a crude version of (1.1), the result

$$
B_{\mathcal{D}}(x)=\frac{6}{\pi^{2}} a(\mathcal{D}) x+O\left(x^{1 / 2} \omega(x)\right),
$$

where

$$
\omega(x)=\exp \left(-c(\log x)^{3 / 5}(\log \log x)^{-1 / 5}\right)
$$

with $c>0$, is a factor familiar from the Prime Number Theorem. (1.5) and (1.6) contain the strongest information available to date concerning zero-free regions of the Riemann zeta-function. At the present state of art, it is not possible to reduce the exponent $1 / 2$ of $x$ in the order term of (1.6). This will be evident from Lemma 1 below (with $y=1$ ), in view of the fact that $\zeta(s)$ could have zeros with real part arbitrarily close to 1 .

It is therefore natural to look for stronger estimates assuming the truth of the Riemann Hypothesis (henceforth quoted as RH). 
In this sense, we want to point out first that, for the case that $\mathcal{D}$ is the unit disk $\mathcal{D}_{0}$, say, a sharp conditional result is essentially contained in the recent literature, namely: Under $\mathrm{RH}$,

$$
B_{\mathcal{D}_{0}}(x)=\frac{6}{\pi} x+O\left(x^{3 / 8+\varepsilon}\right) \quad(\varepsilon>0) .
$$

In fact, Baker [2] studied the summatory function of $d^{(2)}(n)$, the number of square-free (positive) divisors of $n \in \mathbb{N}$, which is generated by $(\zeta(s))^{2} / \zeta(2 s)$. Conditionally under $\mathrm{RH}$, he proved that

$$
\sum_{n \leq x} d^{(2)}(n)=\operatorname{Res}_{s=1}\left(\frac{(\zeta(s))^{2}}{\zeta(2 s)} \frac{x^{s}}{s}\right)+O\left(x^{3 / 8+\varepsilon}\right) \quad(\varepsilon>0) .
$$

Baker used and elaborated a method which has its origin in a well-known paper of Montgomery and Vaughan [15] (who dealt with the distribution of square-free numbers) and has been applied meanwhile to a large class of arithmetic functions: cf. [19] and [20]. Baker's main original ingredient was the use of the truncated Voronoï formula

$$
\begin{aligned}
\sum_{n \leq x} d(n)= & x \log x+(2 \gamma-1) x+\frac{x^{1 / 4}}{\pi \sqrt{2}} \sum_{n \leq N} \frac{d(n)}{n^{3 / 4}} \cos \left(4 \pi \sqrt{n x}-\frac{\pi}{4}\right) \\
& +O\left(x^{1 / 2+\varepsilon} N^{-1 / 2}\right)
\end{aligned}
$$

(where $d(n)$ is the usual divisor function and $N=N(x)=o(x)$ ), along with a deep result due to Heath-Brown [4] to estimate exponential sums which contain the Möbius function.

It is easy to see that Baker's argument can be readily modified to handle $B_{\mathcal{D}_{0}}(x)$ : The corresponding generating function now reads $\zeta_{Q_{0}}(s) / \zeta(2 s)$, where $\zeta_{Q_{0}}$ is the Epstein zeta-function of the quadratic form $Q_{0}\left(u_{1}, u_{2}\right)=$ $u_{1}^{2}+u_{2}^{2}$. One can thus combine the argument in [19], proof of Theorem 1, with Baker's reasoning; an analog of the Voronoï formula, with $d(n)$ replaced by $r(n)$, may be found in Ivić [12], p. 373. Of course, the result may be generalized immediately to the case that $\mathcal{D}$ is a rational ellipse disk, i.e., $Q$ is a positive definite binary quadratic form with rational coefficients.

For general $\mathcal{D}$, however, a perfect extension of this analysis is not in sight, for the following two reasons: On the one hand, the zeta-function of the convex set $\mathcal{D}$ does not satisfy a functional equation as $\zeta_{Q_{0}}$ does. On the other hand, it is not easy to see how to adapt the Fouvry-Iwaniec monomial result for the general situation.

Nevertheless, we shall show here that the core of the MontgomeryVaughan method can be applied to the general problem. Our ultimate goal is to prove the following. 
TheOREM. If $R H$ is true,

$$
B_{\mathcal{D}}(x)=\frac{6}{\pi^{2}} a(\mathcal{D}) x+O\left(x^{5 / 12+\varepsilon}\right),
$$

for a large real variable $x$ and arbitrary fixed $\varepsilon>0$.

The sharpest conditional results of this type known so far is due to Moroz [16], Theorem 1, who obtained the error term $O\left(x^{41 / 91+\varepsilon}\right)$ (if one incorporates Huxley's bound (1.1)). Numerically, 41/91 $=0.450549 \ldots$, while $5 / 12=0.41666 \ldots$ We remark parenthetically that Hensley [5] has recently written a paper on the subject, too, apparently unaware of Moroz's work. His approach was original in method but failed to sharpen the estimate.

It should be emphasized that our result does not depend on the very deep estimate (1.1) but only on an easy version thereof (with the exponent $1 / 3)$, and on the mean-value bound (1.3).

Before going into technical details (which we postpone to Sections 2-4), we outline the essential ideas of the proof. Let

$$
Q_{1}:=\min _{\mathbf{u} \in \mathbb{Z}_{*}^{2}} Q(\mathbf{u}) .
$$

Then it is clear that we may restrict the sum in (1.4) to $1 \leq m \leq \sqrt{x / Q_{1}}$, and split it up as

$$
B_{\mathcal{D}}(x)=\sum_{m \leq y} \mu(m) A_{\mathcal{D}}\left(\frac{x}{m^{2}}\right)+\sum_{m>y} \mu(m) A_{\mathcal{D}}\left(\frac{x}{m^{2}}\right)=: S_{1}+S_{2},
$$

where $y=y(x)<\sqrt{x / Q_{1}}$ is a parameter remaining at our disposition. Let

$$
\begin{aligned}
S_{1} & =a(\mathcal{D}) x \sum_{m \leq y} \frac{\mu(m)}{m^{2}}+\sum_{m \leq y} \mu(m) P_{\mathcal{D}}\left(\frac{x}{m^{2}}\right) \\
& =: a(\mathcal{D}) x \sum_{m \leq y} \frac{\mu(m)}{m^{2}}+S_{1}^{*} .
\end{aligned}
$$

Then the first important step is to prove the following.

Proposition 1. The sum $S_{1}^{*}$ defined in (1.10) satisfies the estimate

$$
S_{1}^{*} \ll y+x^{1 / 4} y^{1 / 2}+x^{5 / 12}
$$

for large real parameters $x$ and $y$ with $1 \leq y \ll x^{1 / 2}$.

To deal with $S_{2}$, an obvious possibility is to use the classic conditional bound (valid under $\mathrm{RH}$ )

$$
\sum_{m \leq Y} \mu(m) \ll Y^{1 / 2+\varepsilon^{\prime}} \quad\left(\varepsilon^{\prime}>0\right) .
$$


Applying summation by parts repeatedly and observing that $A_{\mathcal{D}}(w)$ is monotone and $\ll w$, one obtains

$$
S_{2} \ll x^{\varepsilon^{\prime}} y^{1 / 2} \frac{x}{y^{2}} .
$$

(See Moroz [16], formula (8).)

The second key step of the present paper is to improve this elementary estimate by a contour integration technique in the spirit of Montgomery and Vaughan [15].

Proposition 2. If the Riemann Hypothesis is true,

$$
\begin{aligned}
S_{2} & =\sum_{m>y} \mu(m) A_{\mathcal{D}}\left(\frac{x}{m^{2}}\right) \\
& =a(\mathcal{D}) x \sum_{m>y} \frac{\mu(m)}{m^{2}}+O\left(x^{1 / 3+\varepsilon^{\prime}}\right)+O\left(x^{\varepsilon^{\prime}} y^{1 / 2}\left(\frac{x}{y^{2}}\right)^{3 / 4}\right) \quad\left(\varepsilon^{\prime}>0\right),
\end{aligned}
$$

for large real parameters $x$ and $y$ with $1 \leq y \ll x^{1 / 2}$.

We combine this result with (1.10) and Proposition 1, noting that the two $O$-terms are of the same order (apart from $\varepsilon$ 's) for

$$
y=x^{1 / 3} \text {. }
$$

This choice of $y$ readily yields the assertion of our theorem.

2. Proof of Proposition 1. Since $A_{\mathcal{D}}(w)$ is increasing,

$$
P_{\mathcal{D}}(u)-P_{\mathcal{D}}(v) \geq-a(\mathcal{D})(u-v)
$$

for any positive reals $u \geq v$. Therefore, if $V$ is some positive number and $t>0$ a value for which $\left|P_{\mathcal{D}}(t)\right| \geq V$, there exists an interval $I$ of length $V /(2 a(\mathcal{D}))$ containing $t$ such that

$$
\left|P_{\mathcal{D}}(u)\right| \geq V / 2 \quad \text { for } u \in I .
$$

For positive real parameters $M \ll x^{1 / 2}$ and $V$, let

$$
\begin{aligned}
\mathcal{M}(M, V) & :=\left\{m \in \mathbb{N}, M<m \leq 2 M:\left|P_{\mathcal{D}}\left(x / m^{2}\right)\right| \geq V\right\}, \\
R(M, V) & :=\# \mathcal{M}(M, V) .
\end{aligned}
$$

For any $m \in \mathcal{M}(M, V)$, by the above consideration there exists an interval

$$
J \subseteq\left[\frac{x}{\left(m+\frac{1}{2}\right)^{2}}, \frac{x}{\left(m-\frac{1}{2}\right)^{2}}\right]
$$

of length $|J| \gg \min \left(V, x / M^{3}\right)$, such that $\left|P_{\mathcal{D}}(u)\right| \geq V / 2$ for $u \in J$. As a 
trivial consequence,

$$
V^{2} R(M, V) \min \left(V, \frac{x}{M^{3}}\right) \ll \int_{0}^{x(M-1 / 2)^{-2}}\left(P_{\mathcal{D}}(u)\right)^{2} d u \ll \frac{x^{3 / 2}}{M^{3}},
$$

by an appeal to (1.3). In other words,

$$
R(M, V) \ll \frac{x^{3 / 2}}{M^{3} V^{2}}\left(\frac{1}{V}+\frac{M^{3}}{x}\right)=\frac{x^{3 / 2}}{M^{3} V^{3}}+\frac{x^{1 / 2}}{V^{2}} .
$$

By a familiar device,

$$
\begin{aligned}
\sum_{M<m \leq 2 M}\left|P_{\mathcal{D}}\left(\frac{x}{m^{2}}\right)\right| & \ll M+\sum_{V=2^{j}, j \in \mathbb{N}} V R(M, V) \\
& \ll M+\sum_{V=2^{j}, j \in \mathbb{N}} \min \left(M V, \frac{x^{3 / 2}}{M^{3} V^{2}}+\frac{x^{1 / 2}}{V}\right) \\
& \ll M+x^{1 / 2} M^{-1 / 3}+M^{1 / 2} x^{1 / 4} .
\end{aligned}
$$

Now we let $M$ run through the sequence $y / 2, y / 4, \ldots$ to conclude that

$$
\sum_{\substack{M=y 2^{-j}, j \in \mathbb{N} \\ M>x^{1 / 4}}} \sum_{M<m \leq 2 M}\left|P_{\mathcal{D}}\left(\frac{x}{m^{2}}\right)\right| \ll y+x^{1 / 4} y^{1 / 2}+x^{5 / 12} .
$$

On the other hand, an easy and classic version of (1.1), namely

$$
P_{\mathcal{D}}(w) \ll w^{1 / 3},
$$

implies that

$$
\sum_{m \ll x^{1 / 4}}\left|P_{\mathcal{D}}\left(\frac{x}{m^{2}}\right)\right| \ll x^{1 / 3} \sum_{m \ll x^{1 / 4}} m^{-2 / 3} \ll x^{5 / 12} .
$$

Together with (2.1) this completes the proof of Proposition 1.

3. Some lemmas. The zeta-function $Z_{\mathcal{D}}(s)$ of the convex set $\mathcal{D}$ is defined, for $\operatorname{Re} s>1$, by the absolutely convergent Dirichlet series

$$
Z_{\mathcal{D}}(s)=\sum_{\mathbf{m} \in \mathbb{Z}_{*}^{2}}(Q(\mathbf{m}))^{-s} .
$$

According to Hlawka [8], $Z_{\mathcal{D}}(s)$ possesses an analytic continuation to the whole complex plane, with the exception of one simple pole at $s=1$ with residue $a(\mathcal{D})$.

We define further, for real $y \geq 1$ and a complex variable $s$,

$$
f_{y}(s)=\frac{1}{\zeta(s)}-\sum_{m \leq y} \frac{\mu(m)}{m^{s}} .
$$


This is regular in every $s \in \mathbb{C}$ which is not a zero of the Riemann zetafunction.

LEMma 1. For a large real variable $x$,

$$
\begin{aligned}
S_{2} & =\sum_{m>y} \mu(m) A_{\mathcal{D}}\left(\frac{x}{m^{2}}\right) \\
& =\frac{1}{2 \pi i} \int_{3-i x^{5}}^{3+i x^{5}} Z_{\mathcal{D}}(s) f_{y}(2 s) \frac{x^{s}}{s} d s+O\left(x^{1 / 3+\varepsilon}\right) \quad(\varepsilon>0),
\end{aligned}
$$

uniformly in $1 \leq y \ll \sqrt{x}$.

Proof. This clearly is a type of truncated Perron's formula. It is hard to find an explicit reference in the literature, although the argument runs on familiar lines:

Let us write the values attained by $Q(\mathbf{m})$, as $\mathbf{m}$ runs through $\mathbb{Z}_{*}^{2}$, in form of a strictly increasing sequence $\left(\lambda_{k}\right)_{k \in \mathbb{N}}$. Put further

$$
\mu_{y}(m)= \begin{cases}\mu(m) & \text { if } m>y, \\ 0 & \text { else. }\end{cases}
$$

Then it follows by the homogeneity of $Q$ that, for $\operatorname{Re} s>1$,

$$
Z_{\mathcal{D}}(s) f_{y}(2 s)=\sum_{\mathbf{n} \in \mathbb{Z}_{*}^{2}} \gamma(\mathbf{n})(Q(\mathbf{n}))^{-s}=\sum_{k=1}^{\infty} \alpha_{k} \lambda_{k}^{-s}
$$

with

$$
\gamma(\mathbf{n}):=\sum_{m \mid \mathbf{n}} \mu_{y}(m) \ll\|\mathbf{n}\|^{\varepsilon^{\prime}} \quad\left(\varepsilon^{\prime}>0\right), \quad \alpha_{k}:=\sum_{\mathbf{n}: Q(\mathbf{n})=\lambda_{k}} \gamma(\mathbf{n}) .
$$

Here $m \mid\left(n_{1}, n_{2}\right)$ means that $m \mid \operatorname{gcd}\left(n_{1}, n_{2}\right)$, and $\|\cdot\|$ denotes the Euclidean norm throughout. Obviously,

$$
S_{2}=\sum_{m>y} \mu(m)\left(\sum_{Q(\mathbf{n}) \leq x / m^{2}} 1\right)=\sum_{m, \mathbf{n}: Q(m \mathbf{n}) \leq x} \mu_{y}(m)=\sum_{k: \lambda_{k} \leq x} \alpha_{k} .
$$

It is well known that, for $a>0, a \neq 1$, and $T$ sufficiently large,

$$
\frac{1}{2 \pi i} \int_{-i T}^{3+i T} \frac{a^{s}}{s} d s=\left\{\begin{array}{l}
\chi(a)+O\left(\frac{a^{3}}{T|\log a|}\right), \\
O\left(a^{3}\right),
\end{array}\right.
$$

where $\chi$ is the characteristic function of the interval $] 1, \infty[$. Of this formula, (*) may be found in Apostol [1], p. 243, while (**) is immediate by taking as a path of integration the boundary of the domain $\{s \in \mathbb{C}:|s| \leq T, \operatorname{Re} s \leq 3\}$ if $a>1$, resp., of $\{s \in \mathbb{C}:|s| \leq T$, Re $s \geq 3\}$ if $a<1$ (cf. Prachar [21], p. 379). 
Therefore, by (3.2) and (3.3),

$$
\begin{aligned}
& \frac{1}{2 \pi i} \int_{3-i x^{5}}^{3+i x^{5}} Z_{\mathcal{D}}(s) f_{y}(2 s) \frac{x^{s}}{s} d s \\
& \quad=S_{2}+\sum_{k:\left|\lambda_{k}-x\right| \geq 1} O\left(\frac{\left|\alpha_{k}\right|}{\lambda_{k}^{3} x^{2}\left|\log \lambda_{k}-\log x\right|}\right)+\sum_{k:\left|\lambda_{k}-x\right|<1} O\left(\left|\alpha_{k}\right|\right) .
\end{aligned}
$$

By the mean-value theorem,

$$
\left|\log \lambda_{k}-\log x\right|^{-1} \leq \frac{\max \left(\lambda_{k}, x\right)}{\left|\lambda_{k}-x\right|} \ll \frac{\lambda_{k} x}{\left|\lambda_{k}-x\right|},
$$

thus the first order term sum here is

$$
\ll \frac{1}{x} \sum_{k=1}^{\infty}\left|\alpha_{k}\right| \lambda_{k}^{-2} \ll 1,
$$

since the series in (3.2) converges absolutely for $\operatorname{Re} s>1$. Further,

$$
\left|\alpha_{k}\right| \leq \sum_{\mathbf{n}: Q(\mathbf{n})=\lambda_{k}}|\gamma(\mathbf{n})| \ll \lambda_{k}^{\varepsilon^{\prime}} \sum_{\mathbf{n}: Q(\mathbf{n})=\lambda_{k}} 1,
$$

thus the second order term sum in (3.4) is

$$
\ll x^{\varepsilon^{\prime}} \sum_{\mathbf{n}:|Q(\mathbf{n})-x|<1} 1 \ll x^{\varepsilon^{\prime}}\left(A_{\mathcal{D}}(x+1)-A_{\mathcal{D}}(x-1)\right) \ll x^{1 / 3+\varepsilon^{\prime}},
$$

in view of (2.2). This proves Lemma 1.

The key point to prove Proposition 2 will be to have at hand the following estimates for the growth of the complex function $Z_{\mathcal{D}}(s)$ in the vertical direction.

Lemma 2. (i) For any $\sigma_{1}>1 / 4$ and any $\varepsilon^{\prime}>0$,

$$
Z_{\mathcal{D}}(\sigma+i t) \ll|t|^{(4 / 3)(1-\sigma)+\varepsilon^{\prime}},
$$

uniformly in $\sigma \geq \sigma_{1},|t| \geq 1$.

(ii) For a real parameter $T \geq 1$ and any fixed $\varepsilon^{\prime}>0$,

$$
\int_{T}^{2 T}\left|Z_{\mathcal{D}}\left(\frac{3}{4}+i t\right)\right| d t \ll T^{1+\varepsilon^{\prime}} .
$$

Proof. Let $X$ denote a positive real number which is not attained by $Q(\mathbf{n})$ as $\mathbf{n}$ runs through $\mathbb{Z}_{*}^{2}$. Using Stieltjes integral calculus, we conclude that, for $\operatorname{Re} s>1$, 


$$
\begin{aligned}
& Z_{\mathcal{D}}(s)-\sum_{0<Q(\mathbf{n}) \leq X}(Q(\mathbf{n}))^{-s} \\
& \quad=\int_{X}^{\infty} w^{-s} d\left(a(\mathcal{D}) w+P_{\mathcal{D}}(w)\right) \\
& =a(\mathcal{D}) \int_{X}^{\infty} w^{-s} d w+\int_{X}^{\infty} w^{-s} d P_{\mathcal{D}}(w) \\
& \quad=a(\mathcal{D}) \frac{X^{1-s}}{s-1}-X^{-s} P_{\mathcal{D}}(X)+s \int_{X}^{\infty} w^{-s-1} P_{\mathcal{D}}(w) d w
\end{aligned}
$$

In this identity we choose $0<X<Q_{1}$ and let $X \rightarrow Q_{1}-$ to obtain

$$
Z_{\mathcal{D}}(s)=\frac{a(\mathcal{D}) s}{s-1} Q_{1}^{1-s}+s \int_{Q_{1}}^{\infty} w^{-s-1} P_{\mathcal{D}}(w) d w .
$$

In view of (1.3), this provides an analytic continuation of $Z_{\mathcal{D}}(s)$ to the half-plane $\operatorname{Re} s>1 / 4$ (with the exception of a simple pole at $s=1$ with residue $a(\mathcal{D})$ ), and, at the same time, shows that

$$
Z_{\mathcal{D}}(\sigma+i t) \ll|t|
$$

uniformly in $\sigma \geq \sigma_{1}>1 / 4$ and $|t| \geq 1$. Since, by absolute convergence, $Z_{\mathcal{D}}(\sigma+i t)$ is uniformly bounded in every half-plane $\sigma \geq \sigma_{2}>1$, a Phragmén-Lindelöf argument establishes part (i) of Lemma 2.

To show (ii), we apply the identity derived in (3.5) one more time, with

$$
T^{2} \leq X \leq 2 T^{2}, \quad s=\frac{3}{4}+i t, \quad T \leq t \leq 2 T .
$$

This is clearly justified by analytic continuation. We obtain

$$
\begin{aligned}
& Z_{\mathcal{D}}\left(\frac{3}{4}+i t\right) \\
& \quad=S_{X}(t)+O\left(T^{-1} X^{1 / 4}\right)+O\left(X^{-3 / 4+1 / 3}\right)+O\left(T X^{-1 / 2}\right),
\end{aligned}
$$

with

$$
S_{X}(t):=\sum_{\mathbf{m} \in \mathbb{Z}_{*}^{2}: Q(\mathbf{m}) \leq X}(Q(\mathbf{m}))^{-3 / 4-i t} .
$$

Here we have used (1.3) to estimate the remainder integral in (3.5), and a simple version of (1.1) (with the exponent $1 / 3$ ) to bound $P_{\mathcal{D}}(X)$. Integration over $T \leq t \leq 2 T$ gives

$$
\begin{aligned}
\int_{T}^{2 T}\left|Z_{\mathcal{D}}\left(\frac{3}{4}+i t\right)\right| d t \\
\quad \ll \int_{T}^{2 T}\left|S_{X}(t)\right| d t+O\left(X^{1 / 4}\right)+O\left(T X^{-3 / 4+1 / 3}\right)+O\left(T^{2} X^{-1 / 2}\right) .
\end{aligned}
$$


By Cauchy's inequality $\left({ }^{2}\right)$,

$$
\begin{aligned}
\left(\int_{T}^{2 T}\left|S_{X}(t)\right| d t\right)^{2} & \ll T \int_{T}^{2 T}\left|S_{X}(t)\right|^{2} d t \\
& \ll T \sum_{Q(\mathbf{m}) \leq Q(\mathbf{n}) \leq X}(Q(\mathbf{m}) Q(\mathbf{n}))^{-3 / 4}\left|\int_{T}^{2 T}\left(\frac{Q(\mathbf{n})}{Q(\mathbf{m})}\right)^{i t} d t\right| .
\end{aligned}
$$

For $Q(\mathbf{m})<Q(\mathbf{n})$, the integrals in this sum can be estimated by

$$
\left|\int_{T}^{2 T} \exp (i t(\log Q(\mathbf{n})-\log Q(\mathbf{m}))) d t\right| \leq \frac{2}{\log Q(\mathbf{n})-\log Q(\mathbf{m})} \leq \frac{2 Q(\mathbf{n})}{Q(\mathbf{n})-Q(\mathbf{m})}
$$

Along with the trivial bound, this gives

$$
\begin{aligned}
& \left(\int_{T}^{2 T}\left|S_{X}(t)\right| d t\right)^{2} \\
& \ll T \sum_{Q(\mathbf{n}) \leq X}(Q(\mathbf{n}))^{-3 / 4} \\
& \quad \times\left(\sum_{\mathbf{m}: Q(\mathbf{m}) \leq Q(\mathbf{n})}(Q(\mathbf{m}))^{-3 / 4}\left(\max \left(\frac{1}{T}, \frac{Q(\mathbf{n})-Q(\mathbf{m})}{Q(\mathbf{n})}\right)\right)^{-1}\right) .
\end{aligned}
$$

We now keep $\mathbf{n} \in \mathbb{Z}_{*}^{2}$ fixed for the moment and split up the inner sum over m: First of all,

$$
\frac{1}{T}>\frac{Q(\mathbf{n})-Q(\mathbf{m})}{Q(\mathbf{n})} \Leftrightarrow Q(\mathbf{m})>Q(\mathbf{n})\left(1-\frac{1}{T}\right),
$$

thus the contribution of these $\mathbf{m}$ to the inner sum in (3.8) is

$$
\begin{aligned}
& \ll(Q(\mathbf{n}))^{-3 / 4} T\left(A_{\mathcal{D}}(Q(\mathbf{n}))-A_{\mathcal{D}}\left(Q(\mathbf{n})\left(1-\frac{1}{T}\right)\right)\right) \\
& \ll(Q(\mathbf{n}))^{1 / 4}+T(Q(\mathbf{n}))^{1 / 3-3 / 4},
\end{aligned}
$$

by an easy and classic version of (1.1). Furthermore, we define a sequence $\left(\delta_{j}\right)_{j=0}^{J}$ by $\delta_{j}=2^{j} Q(\mathbf{n}) T^{-1}$, with $J$ such that $\frac{1}{8} Q(\mathbf{n})<\delta_{J} \leq \frac{1}{4} Q(\mathbf{n})$. Then

$$
Q(\mathbf{n})-Q(\mathbf{m}) \in\left[\delta_{j}, 2 \delta_{j}\left[\Leftrightarrow Q(\mathbf{n})-2 \delta_{j}<Q(\mathbf{m}) \leq Q(\mathbf{n})-\delta_{j},\right.\right.
$$

thus the corresponding portion of the inner sum in (3.8) is

$$
\begin{aligned}
& \ll \frac{(Q(\mathbf{n}))^{1 / 4}}{\delta_{j}}\left(A_{\mathcal{D}}\left(Q(\mathbf{n})-\delta_{j}\right)-A_{\mathcal{D}}\left(Q(\mathbf{n})-2 \delta_{j}\right)\right) \\
& \ll(Q(\mathbf{n}))^{1 / 4}+\delta_{j}^{-1}(Q(\mathbf{n}))^{1 / 4+1 / 3} .
\end{aligned}
$$

$\left({ }^{2}\right)$ Here and in what follows, $\mathbf{m}$ and $\mathbf{n}$ denote elements of $\mathbb{Z}_{*}^{2}$. 
Summing this over $j=0, \ldots, J$ gives

$$
\begin{aligned}
& O\left(J(Q(\mathbf{n}))^{1 / 4}\right)+O\left(\delta_{0}^{-1}(Q(\mathbf{n}))^{1 / 4+1 / 3}\right) \\
&=O\left(T^{\varepsilon^{\prime}}(Q(\mathbf{n}))^{1 / 4}\right)+O\left(T(Q(\mathbf{n}))^{1 / 3-3 / 4}\right) .
\end{aligned}
$$

Finally, the portion of the inner sum in (3.8) corresponding to the m's with $Q(\mathbf{n})-Q(\mathbf{m}) \geq 2 \delta_{J}$ is

$$
\ll \sum_{\mathbf{m}: Q(\mathbf{m}) \leq Q(\mathbf{n})}(Q(\mathbf{m}))^{-3 / 4}=\int_{1 / 2}^{Q(\mathbf{n})} u^{-3 / 4} d A_{\mathcal{D}}(u) \ll(Q(\mathbf{n}))^{1 / 4} .
$$

We now combine the upper bounds (3.9)-(3.11), and use them in (3.8) to conclude that

$$
\begin{aligned}
& \left(\int_{T}^{2 T}\left|S_{X}(t)\right| d t\right)^{2} \\
& \quad=T \sum_{Q(\mathbf{n}) \leq X}(Q(\mathbf{n}))^{-3 / 4}\left(O\left(T^{\varepsilon^{\prime}}(Q(\mathbf{n}))^{1 / 4}\right)+O\left(T(Q(\mathbf{n}))^{1 / 3-3 / 4}\right)\right) \\
& \quad=T^{1+\varepsilon^{\prime}} \int_{1 / 2}^{X} w^{-1 / 2} d A_{\mathcal{D}}(w)+O\left(T^{2}\right) \ll T^{1+\varepsilon^{\prime}} X^{1 / 2}+T^{2} .
\end{aligned}
$$

Combining this with (3.7) and recalling that $X \asymp T^{2}$, we complete the proof of Lemma 2.

Lemma 3. If $R H$ is true, the function $f_{y}(s)$ defined in (3.1) satisfies

$$
f_{y}(\sigma+i t) \ll y^{1 / 2-\sigma+\varepsilon^{\prime}}\left(|t|^{\varepsilon^{\prime}}+1\right) \quad\left(\varepsilon^{\prime}>0 \text { fixed }\right),
$$

uniformly in $\sigma_{1} \leq \sigma \leq \sigma_{2}, y \geq 1$, for arbitrary $\sigma_{2}>\sigma_{1}>1 / 2$.

Proof. This key lemma of the Montgomery-Vaughan method is meanwhile well known. See, e.g., Nowak and Schmeier [20], or Baker [2], Lemma 1.

4. Proof of Proposition 2. We start from Lemma 1 and shift the line of integration to $\operatorname{Re} s=3 / 4$, applying the residue theorem. In view of clause (i) of Lemma 2 and Lemma 3 , the horizontal segments contribute

$$
\ll x^{-2} \int_{3 / 4}^{3}\left|Z_{\mathcal{D}}\left(\sigma+i x^{5}\right) f_{y}\left(2 \sigma+2 i x^{5}\right)\right| d \sigma \ll x^{-2+5 \varepsilon^{\prime}+5\left(1 / 3+\varepsilon^{\prime}\right)} \ll 1 .
$$

Furthermore, by clause (ii) of Lemma 2 and Lemma 3, 


$$
\begin{aligned}
& \int_{3 / 4-i x^{5}}^{3 / 4+i x^{5}} Z_{\mathcal{D}}(s) f_{y}(2 s) x^{s} \frac{d s}{s} \\
& \ll x^{3 / 4} y^{1 / 2-3 / 2+\varepsilon^{\prime}}\left(1+\sum_{T=2^{-j} x^{5}, j=1,2, \ldots} T^{\varepsilon^{\prime}-1} \int_{T}^{2 T}\left|Z_{\mathcal{D}}\left(\frac{3}{4}+i t\right)\right| d t\right) \\
& \ll x^{3 / 4+10 \varepsilon^{\prime}} y^{-1+\varepsilon^{\prime}} .
\end{aligned}
$$

Collecting results, we arrive at

$$
S_{2}=\operatorname{Res}_{s=1}\left(Z_{\mathcal{D}}(s) f_{y}(2 s) \frac{x^{s}}{s}\right)+O\left(x^{1 / 3+\varepsilon^{\prime}}\right)+\left(x^{\varepsilon} y^{1 / 2}\left(\frac{x}{y^{2}}\right)^{3 / 4}\right) .
$$

Since this residue is obviously equal to $a(\mathcal{D}) x f_{y}(2)$, this completes the proof of Proposition 2 and thereby that of our theorem.

\section{References}

[1] T. M. Apostol, Introduction to Analytic Number Theory, Springer, New York, 1976.

[2] R. C. Baker, The square-free divisor problem, Quart. J. Math. Oxford 45 (1994), 269-277.

[3] J. L. Hafner, New omega theorems for two classical lattice point problems, Invent. Math. 63 (1981), 181-186.

[4] D. R. Heath-Brown, The Piatetski-Shapiro prime-number theorem, J. Number Theory 16 (1983), 242-266.

[5] D. Hensley, The number of lattice points within a contour and visible from the origin, Pacific J. Math. 166 (1994), 295-304.

[6] E. Hlawka, Über Integrale auf konvexen Körpern I, Monatsh. Math. 54 (1950), $1-36$.

[7] —, Über Integrale auf konvexen Körpern II, ibid., 81-99.

[8] —, Über die Zetafunktion konvexer Körper, ibid., 100-107.

[9] M. N. Huxley, Exponential sums and lattice points II, Proc. London Math. Soc. 66 (1993), 279-301.

[10] _, The mean lattice point discrepancy, Proc. Edinburgh Math. Soc. 38 (1995), 523-531.

[11] - Area, Lattice Points, and Exponential Sums, Oxford University Press, to appear.

[12] A. Ivić, The Riemann Zeta-function, Wiley, New York, 1985.

[13] I. Kátai, The number of lattice points in a circle, Ann. Univ. Sci. Budapest. Eötvös Sect. Math. 8 (1965), 39-60.

[14] E. Krätzel, Lattice Points, Deutsch. Verlag Wiss., Berlin, 1988.

[15] H. L. Montgomery and R. C. Vaughan, The distribution of squarefree numbers, in: Recent Progress in Analytic Number Theory, Proc. Durham Sympos. 1979, Vol. I, H. Halberstam and C. Hooley (eds.), Academic Press, London, 1981, 247-256.

[16] B. Z. Moroz, On the number of primitive lattice points in plane domains, Monatsh. Math. 99 (1985), 37-43. 
[17] W. G. Nowak, An $\Omega$-estimate for the lattice rest of a convex planar domain, Proc. Roy. Soc. Edinburgh Sect. A 100 (1985), 295-299.

[18] - On the average order of the lattice rest of a convex planar domain, Proc. Cambridge Philos. Soc. 98 (1985), 1-4.

[19] - , Primitive lattice points in rational ellipses and related arithmetic functions, Monatsh. Math. 106 (1988), 57-63.

[20] W. G. Nowak and M. Schmeier, Conditional asymptotic formulae for a class of arithmetic functions, Proc. Amer. Math. Soc. 103 (1988), 713-717.

[21] K. Prachar, Primzahlverteilung, Springer, Berlin, 1957.

School of Mathematics

University of Wales

23 Senghenydd Road

Cardiff CF2 4YH

Wales, Great Britain

E-mail: smamnh@cardiff.ac.uk
Institut für Mathematik Universität für Bodenkultur A-1180 Wien, Austria E-mail: nowak@mail.boku.ac.at

Received on 10.7.1995

and in revised form on 19.12.1995 